\title{
Crystal Structure of 9-Benzyl-2-fluoroadenine
}

\author{
Martha M. Rezende*, B. HerberT*, Kenneth L. KirK*, Yuefa GonG** and Hiroshi Kimoto** \\ *National Institute of Diabetes, and Digestive and Kidney Diseases, National Institutes of Health, \\ Bethesda, Maryland 20892, USA \\ **National Industrial Research Institute of Nagoya, Hirate-cho, Kita, Nagoya 462-8510, Japan
}

As a part of our continuing investigations on the biological properties of fluorine-containing heterocyclic compounds, we have been synthesizing novel 2-fluoroadenosine derivatives for evaluation as activators and inhibitors of adenosine-dependent regulatory processes. An extremely useful synthetic intermediate for this purpose is 9-benzyl-2-fluoroadenine. This adenine analog was originally prepared from 2,6-diaminopurine by $N$ benzylation, followed by a Schiemann reaction. ${ }^{1,2}$ The preparation required a critical differentiation between $\mathrm{N}(3)$ and $\mathrm{N}(4)$ during benzylation and between the $\mathrm{C}(1)$ and $\mathrm{C}(2)$ amines during fluorination. The identity of 9benzyl-2-fluoroadenine was reported based on the compound's melting point and the combustion analysis. Although the positional attachment of the benzyl group and the fluorine atom were argued effectively, no concrete evidence was presented which secured the proposed identity. Similar adenine analogs have recently been prepared and have been characterized to a greater extent.

Starting from 2,6-diaminopurine, we prepared 9-benzyl-2,6-diaminopurine and converted it to 9-benzyl-2fluoroadenine by a one-pot diazotization/fluorination reaction. ${ }^{3}$ The melting point of our synthetic compound was identical to that reported. ${ }^{1}$ The ${ }^{1} \mathrm{H},{ }^{13} \mathrm{C}$, and ${ }^{19} \mathrm{~F}$ NMR data were also consistent with related adenine analogs. ${ }^{4}$ Despite the similarity of our compound to these analogs, we could not unequivocally assign the identity of our compound as being the 9-benzyl, 2fluoro analog. Thus, we carried out a single X-ray crystallographic structural analysis that firmly estab-

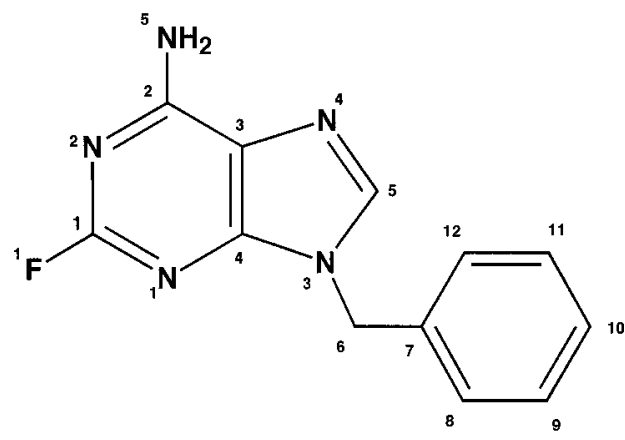

Fig. 1 9-Benzyl-2-fluoroadeine. lishes the structure as 9-benzyl-2-fluoroadenine.

Recrystallization of the synthetic sample from acetone gave colorless plates (mp $272^{\circ} \mathrm{C} \mathrm{dec}$.) which are suitable for X-ray analysis. Of the 5416 reflections measured, 5169 were unique $\left(R_{\text {int }}=0.017\right)$. The crystal data and experimental details are summarized in Table 1 .

The non-H atoms were refined with anisotropic thermal parameters, and the $\mathrm{H}$ atoms were refined with the isotropic ones. The $\mathrm{H}$ atoms bonding to the $\mathrm{C}$ atoms were assigned based on the expected bonding geometry, and other $\mathrm{H}$ atoms were found in a difference Fourier map. The final coordinates of the non-H atoms are given in Table 2; the selected bond lengths and

Table 1 Crystal and experimental data

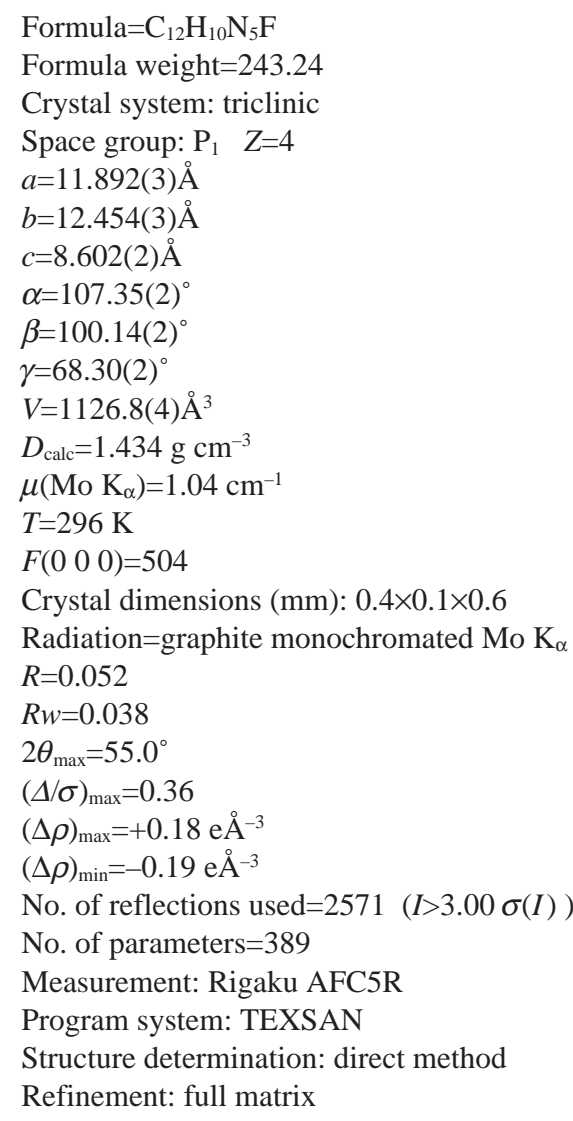

Formula weight $=243.24$

Crystal system: triclinic

$a=11.892(3) \AA$

$b=12.454(3) \AA$

$c=8.602(2) \AA$

$\alpha=107.35(2)$

$\beta=100.14(2)^{\circ}$

$\mu\left(\mathrm{Mo} \mathrm{K}_{\alpha}\right)=1.04 \mathrm{~cm}^{-1}$

$T=296 \mathrm{~K}$

$F(000)=504$

Radiation=graphite monochromated $\mathrm{Mo} \mathrm{K}_{\alpha}$

$R=0.052$

No. of reflections used $=2571(I>3.00 \sigma(I))$

No. of parameters $=389$

Refinement: full matrix 
Table 2 Final atomic coordinates and equivalent isotropic displacement parameters $\left(\AA^{2}\right)$

\begin{tabular}{|c|c|c|c|c|}
\hline Atom & $x$ & $y$ & $z$ & $B_{\text {eq }}$ \\
\hline$F(1)$ & $0.5795(2)$ & $0.8596(2)$ & $-0.0134(2)$ & $5.73(7)$ \\
\hline$F(2)$ & $0.9188(2)$ & $0.1486(2)$ & $-0.3074(2)$ & $4.95(7)$ \\
\hline$N(1)$ & $0.5322(3)$ & $0.8198(3)$ & $0.1986(3)$ & $3.30(8)$ \\
\hline $\mathrm{N}(2)$ & $0.6761(3)$ & $0.9198(3)$ & $0.2223(3)$ & $3.38(8)$ \\
\hline$N(3)$ & $0.5073(3)$ & $0.7987(2)$ & $0.4594(3)$ & $2.98(7)$ \\
\hline$N(4)$ & $0.6380(3)$ & $0.8836(2)$ & $0.6217(3)$ & $2.97(8)$ \\
\hline$N(5)$ & $0.7821(3)$ & $0.9823(3)$ & $0.4637(3)$ & $3.35(8)$ \\
\hline$N(6)$ & $0.9603(3)$ & $0.1944(3)$ & $-0.0375(3)$ & $3.42(8)$ \\
\hline$N(7)$ & $0.8326(3)$ & $0.0763(3)$ & $-0.1728(3)$ & $3.10[8]$ \\
\hline$N(8)$ & $0.9797(3)$ & $0.2221(3)$ & $0.2566(3)$ & $3.22(8)$ \\
\hline $\mathrm{N}(9)$ & $0.8658(3)$ & $0.1182(3)$ & $0.2776(3)$ & $3.29(8)$ \\
\hline $\mathrm{N}(10)$ & $0.7412(3)$ & $-0.0015(3)$ & $-0.0391(3)$ & $3.44(8)$ \\
\hline$C(1)$ & $0.5975(4)$ & $0.8663(3)$ & $0.1485(4)$ & $3.6(1)$ \\
\hline $\mathrm{C}(2)$ & $0.7000[3]$ & $0.9312(3)$ & $0.3858(4)$ & $2,86[9]$ \\
\hline$C(3)$ & $0.6374(3)$ & $0.8855(3)$ & $0.4606(4)$ & $2.60(8)$ \\
\hline$C(4)$ & $0.5575(3)$ & $0.8337(3)$ & $0.3604(4)$ & $2.60(9)$ \\
\hline$C(5)$ & $0.5596(4)$ & $0.8306(3)$ & $0.6154(4)$ & $3.23(10)$ \\
\hline$C(6)$ & $0.4143(4)$ & $0.7399(4)$ & $0.4082(5)$ & $3.5(1)$ \\
\hline$C(7)$ & $0.4670(4)$ & $0.6076(3)$ & $0.3261(4)$ & $3.35(10)$ \\
\hline $\mathrm{C}(8)$ & $0.5769(4)$ & $0.5355(4)$ & $0.3746(6)$ & $4.4(1)$ \\
\hline $\mathrm{C}(9)$ & $0.6180(5)$ & $0.4121(4)$ & $0.2989(7)$ & $5.6(1)$ \\
\hline$C(10)$ & $0.5497(6)$ & $0.3650(5)$ & $0.1710(7)$ & $6.4(2)$ \\
\hline C(11) & $0.4397(6)$ & $0.4364(5)$ & $0.1238(7)$ & $7.3\{2\}$ \\
\hline $\mathrm{C}(12)$ & $0.3978(5)$ & $0.5597(4)$ & $0.1948(5)$ & $5.3(1)$ \\
\hline$C(13)$ & $0.9029(4)$ & $0.1403(3)$ & $-0.1604(4)$ & $3.5(1)$ \\
\hline$C(14)$ & $0.8116(3)$ & $0.0625(3)$ & $-0.0320(4)$ & $2.85(9)$ \\
\hline$C(15)$ & $0.8663(3)$ & $0.1159(3)$ & $0.1152(4)$ & $2.83(9)$ \\
\hline $\mathrm{C}(16)$ & $0.9372(3)$ & $0.1793(3)$ & $0.1005(4)$ & $2.76(9)$ \\
\hline$C(17)$ & $0.9342(4)$ & $0.1818(3)$ & $0.3547(5)$ & $3.47(10)$ \\
\hline$C(18)$ & $1.0655(4)$ & $0.2894(4)$ & $0.3055(5)$ & $4.0(1)$ \\
\hline$C(19)$ & $1.0158(4)$ & $0.4101(3)$ & $0.2700(4)$ & $3.09(9)$ \\
\hline $\mathrm{C}(20)$ & $1.0946(5)$ & $0.4592(4)$ & $0.2407(6)$ & $5.1(1)$ \\
\hline $\mathrm{C}(21)$ & $1.0565(5)$ & $0.5691(5)$ & $0.2126(6)$ & $6.0(1)$ \\
\hline$C(22)$ & $0.9351(6)$ & $0.6348(5)$ & $0.2070(6)$ & $5.6(1)$ \\
\hline$C(23)$ & $0.8559(5)$ & $0.5882(4)$ & $0.2363(6)$ & $5.3(1)$ \\
\hline $\mathrm{C}(24)$ & $0.8955(4)$ & $0.4773(4)$ & $0.2665(5)$ & $4.5(1)$ \\
\hline
\end{tabular}

$B\left({ }_{\mathrm{eq}}\right)=(8 / 3) \pi^{2}\left(U_{11}\left(a a^{*}\right)^{2}+U_{22}\left(b b^{*}\right)^{2}+U_{33}\left(c c^{*}\right)^{2}\right.$

$\left.+2 U_{12} a a^{*} b b^{*} \cos \gamma+2 U_{13} a a^{*} c c^{*} \cos \beta+2 U_{23} b b^{*} c c^{*} \cos \alpha\right)$.

angles are listed in Table 3. An ORTEP drawing and the atomic numbering system of the molecule is shown in Fig. 2.

Although two crystallographically different kinds of molecules were found in the unit cell, we arbitrarily discuss the structure of one representative molecule, because the other one is essentially the same. Their corresponding bond lengths and angles fit within the standard deviations, except for those of the benzene rings, whose atoms have large anisotropic thermal parameters, indicating their high flexibility in the crystal.

The purine ring of the molecule is essentially planar. The $\mathrm{F}(1), \mathrm{N}(5)$, and $\mathrm{C}(6)$ deviate by $-0.017 \AA, 0.025 \AA$ and $-0.031 \AA$, respectively, from the least-squares plane of the purine ring. The dihedral angle between the least-squares planes of the purine and benzene rings is $83.6^{\circ}$.

\section{References}

1. J. A. Montgomery and K. Hewson, J. Am. Chem. Soc., 82, 463 (1960).

2. J. A. Montgomery and K. Hewson, J. Org. Chem., 34, 1396 (1969).
Table 3 Bond distances $(\AA)$ and angles $\left({ }^{\circ}\right)$

\begin{tabular}{|c|c|c|c|c|c|c|c|}
\hline$C(1)$ & $N(1)$ & & $1.312(4)$ & $\mathrm{C}(1)$ & $N(2)$ & & $1.312(4)$ \\
\hline $\mathrm{C}(2)$ & $\mathrm{N}(2)$ & & $1.360(4)$ & $\mathrm{C}(2)$ & $\mathrm{C}(3)$ & & $1.411(4)$ \\
\hline$C(3)$ & $\mathrm{C}(4)$ & & $1.377(4)$ & $\mathrm{C}(4)$ & $\mathrm{N}(1)$ & & $1.343(4)$ \\
\hline $\mathrm{C}(3)$ & $\mathrm{N}(4)$ & & $1.392(4)$ & $C(4)$ & $\mathrm{N}(3)$ & & $1.366(4)$ \\
\hline$C(5)$ & $\mathrm{N}(3)$ & & $1.384(4)$ & $C(5)$ & $\mathrm{N}(4)$ & & $1.313(4)$ \\
\hline$C(1)$ & $\mathbf{F}(1)$ & & $1.353(4)$ & $C(2)$ & $\mathrm{N}(5)$ & & $1.336(4)$ \\
\hline$C(6)$ & $\mathrm{N}(3)$ & & $1.478(4)$ & $C(13)$ & $F(2)$ & & $1.350(4)$ \\
\hline$C(13)$ & $N(6)$ & & $1.303(4)$ & $\mathrm{C}(13)$ & $\mathrm{N}(7)$ & & $1.326(4)$ \\
\hline$C(14)$ & $\mathrm{N}(7)$ & & $1.349(4)$ & $C(14)$ & $\mathrm{C}(15)$ & & $1.413(4)$ \\
\hline $\mathrm{C}(15)$ & $\mathrm{C}(16)$ & & $1.393(4)$ & $\mathrm{C}(16)$ & $N(6)$ & & $1.343(4)$ \\
\hline $\mathrm{C}(15)$ & $N(9)$ & & $1.390(4)$ & $\mathrm{C}(16)$ & $\mathrm{N}(8)$ & & $1.370(4)$ \\
\hline$C(17)$ & $N(8)$ & & $1.368(4)$ & $\mathrm{C}(17)$ & $\mathrm{N}(9)$ & & $1.305(4)$ \\
\hline$C(14)$ & $\mathrm{N}(10)$ & & $1.337(4)$ & $\mathrm{C}(18)$ & $N(8)$ & & $1.480(4)$ \\
\hline $\mathrm{N}(1)$ & $\mathrm{C}(1)$ & $\mathrm{N}(2)$ & $133.7(3)$ & $\mathrm{C}(1)$ & $\mathrm{N}(2)$ & $\mathrm{C}(2)$ & $116.7(3)$ \\
\hline$N(2)$ & $\mathrm{C}(2)$ & $\mathrm{C}(3)$ & $117.2(3)$ & $\mathrm{C}(2)$ & $\mathrm{C}(3)$ & $\mathrm{C}(4)$ & $116.6(3)$ \\
\hline $\mathrm{C}(3)$ & $C(4)$ & $N(1)$ & $128.0(3)$ & $\mathrm{C}(1)$ & $N(1)$ & $\mathrm{C}(4)$ & $107.8(3)$ \\
\hline$C(4)$ & $C(3)$ & $\mathrm{N}(4)$ & $111.0(3)$ & $\mathrm{C}(3)$ & $\mathrm{N}(4)$ & $C(5)$ & $104.0(3)$ \\
\hline$N(3)$ & $C(5)$ & $\mathrm{N}(4)$ & $112.9(3)$ & $C(4)$ & $\mathrm{N}(3)$ & $C(5)$ & $106.3(3)$ \\
\hline $\mathrm{C}(3)$ & $C(4)$ & $\mathrm{N}(3)$ & $105.9(3)$ & $\mathrm{N}(1)$ & $\mathrm{C}(1)$ & $F(1)$ & $114.0(3)$ \\
\hline $\mathrm{N}(2)$ & $C(2)$ & $\mathrm{N}(5)$ & $118.1(3)$ & $C(4)$ & $N(3)$ & $C(6)$ & $126.2(3)$ \\
\hline $\mathrm{N}(3)$ & $C(6)$ & $C(7)$ & $112.8(3)$ & & & & \\
\hline $\mathrm{N}(6)$ & $C(13)$ & $N(7)$ & $133.5(3)$ & $C(13)$ & $N(7)$ & $C(14)$ & $116.5(3)$ \\
\hline $\mathrm{N}(7)$ & $C(14)$ & $C(15)$ & $118.1(3)$ & $C(14)$ & $C(15)$ & $C(16)$ & $116.0(3)$ \\
\hline$C(15)$ & $C(16)$ & $N(6)$ & $127.4(3)$ & $C(13)$ & $\mathrm{N}(6)$ & $C(16)$ & $108.5(3)$ \\
\hline $\mathrm{C}(16)$ & C(15) & $N(9)$ & $110.8(3)$ & $C(15)$ & $N(9)$ & $\mathrm{C}(17)$ & $103.3(3)$ \\
\hline $\mathrm{N}(8)$ & $\mathrm{C}(17)$ & $N(9)$ & $114.6(3)$ & $\mathrm{C}(16)$ & $N(8)$ & $C(17)$ & $105.9(3)$ \\
\hline$C(15)$ & $C(16)$ & $\mathrm{N}(8)$ & $105.4(3)$ & $N(6)$ & $\mathrm{C}(13)$ & $\mathrm{F}(2)$ & $114.4(3)$ \\
\hline$N(7)$ & $\mathrm{C}(14)$ & $\mathrm{N}(10)$ & $118.4(3)$ & $C(16)$ & $N(8)$ & $\mathrm{C}(18)$ & $126.5(3)$ \\
\hline $\mathrm{N}(8)$ & $\mathrm{C}(18)$ & $\mathrm{C}(19)$ & $113.8(3)$ & & & & \\
\hline
\end{tabular}

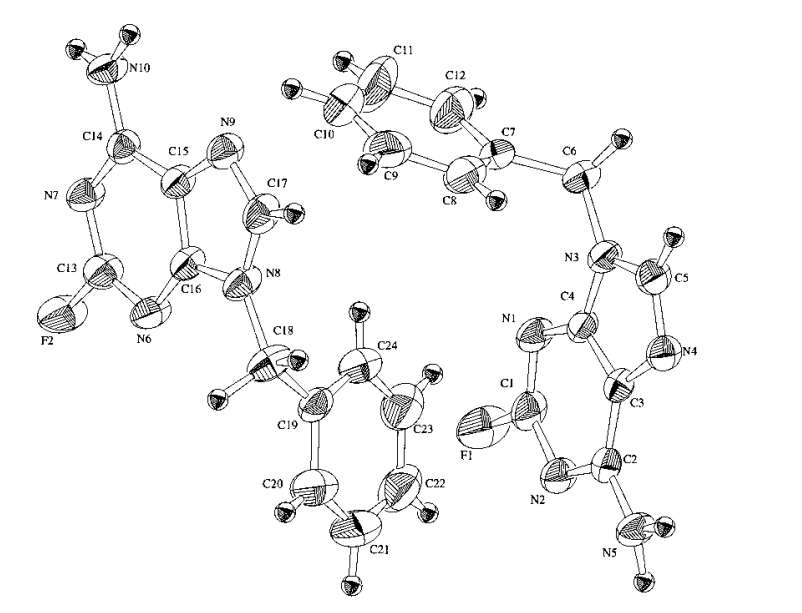

Fig. 2 ORTEP drawing of the molecule and atom numbering scheme.

3. M. M. Rezende, A. B. de Oliveira, B. Herbert and K. L. Kirk, manuscript in preparation.

4. a) N. Platzer, H. Galons, Y. Bensaid, M. Miocque and G. Bram, Tetrahedron, 43, 2101 (1987). b) M. C. Thorpe, W. C. Jr. Coburn and J. A. Montgomery, J. Magn. Reson., 15, 98 (1974). c) W. C. Jr. Coburn, M. C. Thorpe and J. A. Montgomery, J. Org. Chem., 30, 1110 (1965). d) W. C. Jr. Coburn, M. C. Thorpe and J. A. Montgomery, J. Org. Chem., 30, 1114 (1965). e) M. J. Robins and B. Uznanski, Can. J. Chem., 59, 2608 (1981).

(Received March 9, 1999) (Accepted June 7, 1999) 